BULL. AUSTRAL. MATH. SOC.

VOL. 22 (1980), 161-198.
$54 F 40,55-02,55 C 30,54 F 53$

(55P55, 57N25, 55Q07)

\title{
WHAT IS THE THEORY OF SHAPE?
}

\author{
KaROL BORsuK AND JeRzY DYDAK
}

\begin{abstract}
This expository article on Shape Theory contains the main concepts of this theory with a formulation of the most important results of this theory and also with some open problems. The proofs are omitted, however the article gives references to the books and papers, in which the reader can find the proofs. For simplicity, we formulate several theorems only in the form which clearly gives their geometric sense, even if there are known more general results with rather complicated formulations. The reader more interested in details, can find them in the original papers quoted in the list of references.
\end{abstract}

\section{Introduction}

The relationships between the concepts of general topology and geometric intuition constitute the subject called geometric topology, which deals as well with set-theoretical and algebraic methods. Aleksandrov in his talk opening the International Topology Conference in Moscow in 1979, distinguished three periods in the development of this branch of topology (see [1], p. 4; compare also [2] and [3], pp. 33 and 35). The first of these periods is dominated by the works of L.E.J. Brouwer (years 1909-1913). The second period is marked by the development of the theory of homology and cohomology (including the duality theorems), the creation of the theory of dimension and the development of the theory of continua. The third period (which contimues even today) began (according to Aleksandrov) with the creation of the theory of retracts, including the theory of homotopy

Received 6 February 1980. 
groups due to $W$. Hurewicz, and it is continued by the development of the theory of shape.

The aim of this article is to give a short survey of the most important notions and results of the theory of shape, together with some of its problems. We assume that the reader is familiar with the mostly elementary topological notions. We recall also several more special topological concepts, together with indications where the reader can find their exact definitions.

By a map $f: X \rightarrow Y$ of a space $X$ into another space $Y$ we understand always a continuous function. Two maps $f, g: X \rightarrow Y$ are said to be homotopic in a space $Z$ (notation: $f \simeq g$ in $Z$ ) if there exists a $\operatorname{map} \varphi: X \times\langle 0,1\rangle \rightarrow Z$ (called a homotopy) such that $\varphi(x, 0)=f(x)$ and $\varphi(x, 1)=g(x)$ for every $x \in X$. In the case $z=Y$ one says that $f, g: X \rightarrow Y$ are homotopic (notation: $f \simeq g$ ). If there exist maps $f: X \rightarrow Y$ and $g: Y \rightarrow X$ such that $g f \simeq i_{X}$ (where $i_{X}$ denotes the identity map of $X$ ), then one says that $Y$ homotopically dominates $X$. If there exist two maps $f: X \rightarrow Y$ and $g: Y \rightarrow X$ such that $g f \simeq i_{X}$ and $f g \simeq i_{Y}$, then one says that the spaces $X$ and $Y$ are homotopically equivalent, or that they have the same homotopy type. The properties of a space $X$ depending only on its homotopy type are said to be homotopy properties of $X$. By compacta we understand always compact metrizable spaces.

We need also the following concepts:

connectedness in dimension $n$ and local connectedness in dimension $n$ (see, for instance, [10], p. 30); contractibility and local contractibility ([10], pp. 26 and 28); polyhedra (finite), polytopes and their triangulations (see [10], p. 11 and p. 71);

dimension (the covering dimension) (see [47], p. 54); upper semi-continuous decomposition and its quotient space (see [71], p. 64).

Homology group $H_{n}(X, \underline{\underline{A}})$ (over an abelian group $\underline{\underline{A}}$ ) in the sense of 
L. Vietoris, or of E. Čech (see [10], p. 35). If $\underline{\underline{A}}$ is the group of integers, then $H_{n}(X, \underline{\underline{A}})$ is called the $n$th Betti group of $X$ (notation: $H_{n}(X)$ ) and its rank is the $n$th Betti number of $X$ (notation: $p_{n}(X)$ ). If all homology groups $H_{n}(X, \underline{A})$ are trivial, then $X$ is said to be acyclic.

Homotopy groups $\pi_{n}\left(X, x_{0}\right)$ of a pointed, arcwise connected compactum $\left(X, x_{0}\right)$ (see, for instance, [10], p. 50).

\section{Some information on the theory of retracts}

Since the theory of shape is intimately related to the theory of retracts, let us recall some basic notions and results of this latter theory.

A map $f: X+Y$ of a space $X$ into another space $Y$ is said to be an r-map, if there exists for $f$ a right inverse map; that is, a map $g: Y \rightarrow X$ such that $f g: Y \rightarrow Y$ is the identity map $i_{Y}$ for $Y$, that is $i_{Y}(y)=y$ for every $y \in Y$. If there exists an $r$-map of $X$ into $Y$, then we say that $Y$ is an r-image of $X$.

A special kind of $r$-map is a retraction; that is, a map $f: X \rightarrow Y$, where $Y \subset X$ and $f(y)=y$ for every $y \in Y$. If there exists a retraction $f: X \rightarrow Y$, then $Y$ is said to be a retract of $X$. It is known that every retract of a space $X$ is closed in $X$.

In the theory of retracts one distinguishes the following classes of spaces with especially regular properties.

A metrizable space $Y$ is said to be an absolute retract for metrizable spaces (notation: $Y \in \mathrm{AR}(\underline{\underline{M}})$, where $\underline{\underline{M}}$ denotes the class of all metrizable spaces) if for every space $X \in \underline{\underline{M}}$ which contains $Y$ as a closed subset, $Y$ is a retract of $X$. It is well known that every convex subset of any linear normed space is an $\mathrm{AR}(\underline{\underline{M}})$.

A metrizable space $Y$ is said to be an absolute neighborhood retract for metrizable spaces (notation: $Y \in \operatorname{ANR}(\underline{\underline{M}})$ ) if for every space $X \in \underline{\underline{M}}$ containing $Y$ as a closed subset, $Y$ is a retract of some of its neighborhoods in $X$. 
Compact $\mathrm{AR}(\underline{\underline{M}})$ spaces are said to be absolute retracts (notation: $X \in \mathrm{Ak}$ ), and compact $\mathrm{ANR}(\underline{\underline{M}})$ are said to be absolute neighborhood retracts (notation: $X \in$ ANR ). In particular, the Hilbert cube (which we still denote by $Q$ ) is an AR and every polyhedron is an ANR . Clearly every AR-set is contractible in itself to a point.

Many topological properties of polyhedra hold true for all ANR's . So it is, in particular, with all homotopy properties of polyhedra, as it follows from a theorem recently proved by West ([99], p. 13) that every ANR-space has the homotopy type of a polyhedron. However, there exist among ANR's (even among AR's ) spaces with properties very different from the properties of polyhedra. In particular, there exists a 2-dimensional AR-set $A$ such that every non-empty and different from $A$ compactum $X \subset A$, containing a non-empty open subset of $A$, has a positive first Betti number $p_{I}(X)$.

Let us mention some theorems on ANR-sets, which will be useful in the sequel.

THEOREM (2.1) on exiension of a homotopy ([10], p. 94). Let $X$ be a closed subset of a space $X^{\prime} \in \underline{\underline{M}}$ and $Y \in \operatorname{ANR}(\underline{\underline{M}})$. If $\varphi: X \times\langle 0,1\rangle \rightarrow Y$ is a homotopy such that the map $f: X+Y$ given by the formula $f(x)=\varphi(x, 0)$ for every $x \in X$ has an extension to a map $f^{\prime}: X^{\prime} \rightarrow Y$, then there is a homotopy $\varphi^{\prime}: X^{\prime} \times(0,1) \rightarrow Y$ which is an extension of the homotopy $\varphi$ and $\varphi^{\prime}(x, 0)=f^{\prime}(x)$ for every $x \in X^{\prime}$.

THEOREM (2.2) ([10], p. 122). A finite-dimensional compactum is an ANR-space if and only if it is locally contractible.

However there exist infinite-dimensional, locally contractible compacta which are not ANR's ([10], p. 124).

THEOREM (2.3) (Hanner [53], p. 143 and p. 392). Every open subset of a space $X \in \mathrm{ANR}(\underline{\underline{M}})$ is an $\operatorname{ANR}(\underline{\underline{M}})$-space. If for every point $x \in X \in \underline{M}$ there is a neighborhood $U \in \operatorname{ANR}(\underline{\underline{M}})$, then $X \in \operatorname{ANR}(\underline{\underline{M}})$.

It follows that the property to be an $\operatorname{ANR}(\underline{\underline{M}})$-space is a local property.

THEOREM (2.4) (W. Hurewicz; see, for instance, [10], p. 52). If $X$ is a connected ANR-space and $x_{0} \in X$ and if the homotopy groups 
$\pi_{k}\left(X, x_{0}\right)$ are trivial for $k=1,2, \ldots, n-1$, then the group $\pi_{n}\left(X, x_{0}\right)$ is isomorphic to the nth Betti group ${ }_{n}(X)$.

THEOREM (2.5) (Whitehead, [101], p. 1133). If $\left(x, x_{0}\right)$ and $\left(y, y_{0}\right)$ are two connected pointed ANR-spaces and if a map $f:\left(x, x_{0}\right) \rightarrow\left(y, y_{0}\right)$ induces an isomorphism $f_{*, n}: \pi_{n}\left(x, x_{0}\right) \rightarrow \pi_{n}\left(y, y_{0}\right)$ for every $n=1,2, \ldots$, then $f$ is a homotopy equivalence.

THEOREM (2.6) (S. Smale; see, for instance, [10], p. 127). If $f$ is a map of a space $X \in \mathrm{ANR}$ onto a space $Y$ such that $f^{-1}(y) \in \mathrm{AR}$ for every $y \in Y$, then $Y$ is locally n-connected compactum for $n=1,2, \ldots$.

THEOREM (2.7) (K. Kuratowski and M. Wojdysławski; see, for instance, [10], p. 79). Every metrizable space is homeomorphic to a closed subset of a convex, bounded set lying in a Banach space.

\section{Preliminary remarks on the theory of shape}

In the classical homotopy theory only the homotopy properties of spaces are studied. The classification of spaces into homotopy types is based on the study of families of maps of one space into another space. However the local complications in the topological structure of a space $y$ may imply that the family of maps of another space $X$ into $Y$ can be very limited and does not give a sufficient base to study global properties of those spaces. For instance, if $X$ is an arcwise connected space and $Y$ does not contain any arc, then the only maps of $X$ into $Y$ are maps of $X$ onto singletons. Thus the natural domain of the classical homotopy theory are spaces with a rather regular local structure, in particular, the spaces $\operatorname{ANR}(\underline{M})$.

The aim of the theory of shape is to study the global properties of spaces, neglecting the complications in their local structures. In the case of compacta, one gets such an approach to the study of global properties if one replaces in the basic notations of the classical homotopy theory, maps by a more elastic concept of fundamental sequences.

The theory of shape of compacta was initiated in 1968 (see [11]). Later the range of this theory was extended to more general classes of 
spaces. In 1971, Holsztyński ([55]) gave an axiomatic approach to this theory, based on the concepts of the theory of category and exhibiting a deep connection between the concept of shape and the concept of the inverse limit. A little later, Mardešic and Segal (see [78]) gave a systematic tyeory of shape of compact Hausdorff spaces, based on the notion of the inverse system of ANR-spaces. Finally, in 1973, Mardešić (see [73]) extended the notion of the shape to arbitrary topological spaces. Today the commonly used definition of shape category is that one given by Morita [82].

Now the theory of shape is a large branch of topology, and there exist in the world literature more than 400 papers devoted to this theory, among them several synthetic courses of this theory (see [12], [14], [15], [43]).

The most developed is the theory of shape of compacta, which is intimately connected with geometric intuition. However even this part of the theory of shape still contains many open problems.

\section{Fundamental sequences and the concept of the shape of a compactum}

It is well known (see [10], p. 79) that for every compactum $X$ there exist AR-spaces $M$ such that $X \subset M$. Let $Y$ be another compactum and $Y \subset N \in A R$. By a fundomental sequence $f: X+Y$ one understands a sequence of maps $f_{k}: M \rightarrow M, k=1,2, \ldots$, such that

for every neighborhood $V$ of $Y$ in $M$ there is a neighborhood $U$ of $X$ in $M$ such that $f_{k} / U \simeq f_{k+1} / U$ in $V$ for almost all $k$.

Let us denote this fundamental sequence $f$ by $\left\{f_{k}, X, Y\right\}_{M, N} \cdot$ In particular, if $X=Y$ and $M=N$, then setting $f_{k}=i_{M}$ (where $i_{M}$ denotes the identity map of $M$ ) for every $k=1,2, \ldots$, one gets a fundamental sequence $i_{X, M}=\left\{i_{M}, X, X\right\}_{M, M}$ called the fundomental identity sequence for $X$ in $M$.

$$
\text { If } f=\left\{f_{k}, X, Y\right\}_{M, N} \text { and } g=\left\{g_{k}, Y, z\right\}_{N, R} \quad \text { (where } z \subset R \in \mathrm{AR} \text { ), }
$$

then $\mathrm{gf}=\left\{g_{k} f_{k}, X, 2\right\}_{M, R}$ is a fundamental sequence called the composition of $f$ and $g$. 
Two fundamental sequences $f=\left\{f_{k}, X, Y\right\}_{M, N}$ and $f^{\prime}=\left\{f_{k}^{\prime}, X, Y\right\}_{M, N}$ are said to be homotopic (notation: $f \simeq f^{\prime}$ ) if for every neighborhood $V$ of $Y$ in $N$ there is a neighborhood $U$ of $X$ in $M$ such that

$$
f_{k} / U \simeq f_{k}^{\prime} / U \text { in } V \text { for almost all } k \text {. }
$$

One says that a compactum $X \subset M \in \mathrm{AR}$ shape dominates another compactum $Y \subset N \in A R$ if there exist two fundamental sequences $f: X \rightarrow Y$ and $g: Y \rightarrow X$ such that $f g \simeq i_{Y, N}$. If $M, N, f$ and $g$ may be selected so that also $g f \simeq \mathbf{i}_{X, M}$, then one says that $X$ and $Y$ are shape-equivalent. One sees easily that the choice of the spaces $M, N \in \mathrm{AR}$ is immaterial. Thus the shape of a given compactum $X$ (notation: $\operatorname{Sh}(X)$ ) may be considered as the collection of all compacta which are shape-equivalent to $X$.

If a compactum $X$ shape dominates another compactum $Y$, then we write $\operatorname{Sh}(X) \geq \operatorname{Sh}(Y)$. If $\operatorname{Sh}(X) \geq \operatorname{Sh}(Y)$, but $\operatorname{Sh}(X)$ is different from $\operatorname{Sh}(Y)$, then we write $\operatorname{Sh}(X)>\operatorname{Sh}(Y)$. The shape of a singleton is said to be trivial and we denote it by $\operatorname{Sh}(1)$. It is clear that $\operatorname{Sh}(1) \leq \operatorname{Sh}(Y)$ for every compactum $Y \neq \varnothing$. One sees easily that all compacta with trivial shape are acyclic. However, there exist acyclic compacta with nontrivial shape. Such an example is the curve constructed by Case and Chamberlin (see [25]). A simple characterization of compacta with trivial shape is given by the following

THEOREM (4.1) (see [14], p. 262). A compactum $X \neq \emptyset$ has trivial shape if and only if for every space $M \in \operatorname{ANR}(\underline{\underline{M}})$ containing $X$, the set $X$ is contractible to a point in every neighborhood $U$ of $X$ in $M$.

Let us add, that a compactum $X$ lying in the plane $E^{2}$ has trivial shape if $X$ and $E^{2} \backslash X$ are connected.

By a pointed compactum $\left(X, x_{0}\right)$ one understands a compactum $X$ in which a point $x_{0}$ is selected. Let $X \subset M, Y \subset N, M, N \in \mathrm{AR}$ and let $x_{0} \in X, y_{0} \in Y$. If in the definition of the fundamental sequence $\left\{f_{k}, X, Y\right\}_{M, N}$ one considers only maps $f_{k}: M \rightarrow N$ satisfying the condition $f_{k}\left(x_{0}\right)=y_{0}$ for $k=1,2, \ldots$ (notation: 
$\left.f_{k}:\left(M, x_{0}\right) \rightarrow\left(N, y_{0}\right)\right)$ and if one assumes that by homotopies $f_{k} / U \simeq f_{k+1} / U$ in $V$ the point $x_{0}$ is still mapped onto $y_{0}$, then we obtain the notion of the pointed fundamental sequence f : $\left(X, x_{0}\right) \rightarrow\left(y, y_{0}\right)$, which one denotes by $\left\{f_{k},\left(x, x_{0}\right),\left(y, y_{0}\right)\right\}_{M, N}$. It is clear how the notion of the homotopy of two fundamental sequences transfers to the case of pointed compacta, and so it is also with the notions of homotopy domination, of homotopy equivalence and of shape. Thus one gets the concept of pointed shape $\operatorname{Sh}\left(X, x_{0}\right)$ and also of the relations $\operatorname{Sh}\left(X, x_{0}\right) \geq \operatorname{Sh}\left(Y, y_{0}\right)$ and $\operatorname{Sh}\left(X, x_{0}\right)>\operatorname{Sh}\left(y, y_{0}\right)$.

One proves easily that if $X, Y$ are two ANR's, then the relation $\operatorname{Sh}(X)=\operatorname{Sh}(Y)$ holds true if and only if the homotopy types of $X$ and of $Y$ coincide, and the relation $\operatorname{Sh}(X) \geq \operatorname{Sh}(Y)$ is equivalent to the homotopy domination of $X$ over $Y$. Thus in the domain of ANR-spaces the concepts of the theory of shape do not exceed the range of the corresponding homotopy concepts. The situation is quite different for arbitrary compacta, as seen by the following

THEOREM (4.2) (see [14], p. 221 and p. 222). If $X, Y$ are two plane continua, then $\operatorname{Sh}(X)=\operatorname{Sh}(Y)$ if and only if $X$ and $Y$ decompose the plane $E^{2}$ into the same number of regions, and $\operatorname{Sh}(X) \geq \operatorname{Sh}(Y)$ if and only if the number of components of $E^{2} \backslash X$ is greater than or equal to the number of components of $E^{2} \backslash Y$.

It follows that for shapes of plane continua the low of the trichotomy holds true (that is, if $X, Y$ are plane continua, then either $\operatorname{Sh}(X)=\operatorname{Sh}(Y)$, or $\operatorname{Sh}(X)>\operatorname{Sh}(Y)$, or $\operatorname{Sh}(X)<\operatorname{Sh}(Y))$.

Another situation prevails for continua lying in the 3-dimensional euclidean space $E^{3}$. There exist continua $X, Y \subset E^{3}$ such that $\operatorname{Sh}(X) \geq \operatorname{Sh}(Y)$ and $\operatorname{Sh}(X) \leq \operatorname{Sh}(Y)$, but $\operatorname{Sh}(X) \neq \operatorname{Sh}(Y)$. The question if such a situation is possible for polyhedra remains open.

By Theorem (4.2), the family of all shapes of plane continua is countable. One proves easily that there exist $2^{K_{0}}$ different shapes of plane compacta. By a theorem due to Spiez ([94], p. 155) there exists a 
plane compactum $X_{0}$ such that for every plane compactum $X \neq \varnothing$, $\operatorname{Sh}(X) \leq \operatorname{Sh}\left(X_{0}\right)$. It is not so for compacta lying in $E^{3}$, because already the family consisting of all solenoids of van Dantzig (concerning this notion see [35], p. 106) there does not exist any compactum with shape greater than or equal to the shapes of all those solenoids (see [22], p. 108).

Let us denote by $\square(X)$, for every compactum $X$, the decomposition space of the decomposition of $X$ into its components. The following theorem holds true:

THEOREM (4.3) (see [14], p. 214). Let $X, Y$ be two compacta, $X \subset M, \quad Y \subset N, M, N \in \mathrm{AR}$. For every fundamental sequence $f=\left\{f_{k}, X, Y\right\}_{M, N}$ there exists a map

$$
\Lambda_{f}: \square(X) \rightarrow \square(Y)
$$

such that for every component $X_{0}$ of $X$ the sequence $\left\{f_{k} X_{0}, \Lambda_{f}\left(X_{0}\right)\right\}_{M, N}$ is fundamental. Moreover, if $Z$ is a compactum lying in a space $R \in \mathrm{AR}$, and if $\mathrm{g}=\left\{g_{k}, Y, Z\right\}_{N, R}$ is a fundamental sequence, then $\Lambda_{\mathrm{gf}}=\Lambda_{\mathrm{g}} \Lambda_{\mathrm{f}}$.

It follows by this theorem that

If $X, Y$ are compacta with $\mathrm{Sh}(X) \geq \mathrm{Sh}(Y)$, then there exists an r-map $\Lambda: \square(X) \rightarrow \square(Y)$ such that $\operatorname{Sh}\left(X_{0}\right) \geq \operatorname{Sh}\left(\Lambda\left(X_{0}\right)\right)$ for every $x_{0} \in \square(X)$. Moreover, if $\operatorname{Sh}(X)=\operatorname{Sh}(Y)$, then there exists a homeomorphism $\Lambda: \square(X) \underset{\text { onto }}{\longrightarrow} \square(Y)$ assigning to every component $X_{0}$ of $X$ the component $\Lambda\left(X_{0}\right)$ such that $\operatorname{Sh}\left(X_{0}\right)=\operatorname{Sh}\left(\Lambda\left(X_{0}\right)\right)$.

It follows in particular, that two o-dimensional compacta with the same shape are homeomorphic.

The Hilbert cube $Q$ may be considered as the subset of the Hilbert space consisting of all points $\left(x_{1}, x_{2}, \ldots\right)$ with $0 \leq x_{k} \leq 1 / k$ for $k=1,2, \ldots$. Let us denote by $\dot{Q}$ the so called pseudo-interior of $Q$, defined as the set of all points $\left(x_{1}, x_{2}, \ldots\right)$ with $0<x_{k}<1 / k$ for $k=1,2, \ldots$. One of the most remarkable theorems of the theory of shape is the following 


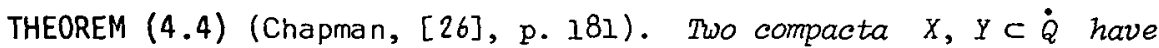
the same shape if and only if the sets $Q \backslash X$ and $Q \backslash Y$ are homeomorphic.

Another theorem of a similar kind characterizes the shape of finite dimensional compacta:

THEOREM (4.5) (see [27], p. 261 and also [50], p. 281). If $X$ and $Y$ are compacta of dimension less than or equal to $k$, lying in the $n$-dimensional euclidean space $E^{n}$ with $n \geq 2 k+2$, and satisfying some additional conditions (which, in particular, hold true for all geometric polyhedra), then $\operatorname{Sh}(X)=\operatorname{Sh}(Y)$ if and only if the sets $E^{n} \backslash X$ and $E^{n} \backslash Y$ are homeomorphic.

\section{Shapes of topological spaces}

In order to define the shape category for topological spaces, we need the notion of the pro-category of a given category, introduced by Grothendieck [51] in a greater generality than we need here. Recall that a category is a triple $C$ consisting of:

1. a class of objects denoted by $\mathrm{Ob}(\mathrm{C})$,

2. a set of morphisms $C(X, Y)$ from $X$ to $Y$ for any two objects $X$ and $Y$ of $\mathrm{C}$,

3. a function (called composition) from $\mathcal{C}(X, Y) \times \mathcal{C}(Y, Z)$ to $\mathcal{C}(X, Z)$ (the image of a pair $(f, g)$ of morphisms is denoted by $g f)$,

such that the following conditions are satisfied:

4. for any morphisms $f \in \mathcal{C}(X, Y), g \in \mathcal{C}(Y, Z)$ and $h \in \mathcal{C}(Z, W)$ there is $h(g f)=(h g) f$,

5. for any object $X$ of $C$ there exists an identity morphism id $_{X} \in \mathcal{C}(X, X)$ such that for any morphisms $g \in \mathcal{C}(X, Y)$ and $h \in \mathcal{C}(Z, X), g\left(\mathrm{id}_{X}\right)=g$ and $\left(i d_{X}\right) h=h$.

A category $D$ is called a full subcategory of a category $C$ provided $\mathrm{Ob}(D)$ is a subclass of $\mathrm{Ob}(\mathcal{C}), D(X, Y)=\mathcal{C}(X, Y)$ for $X, Y \in \mathrm{Ob}(D)$ and the compositions of two morphisms in $D$ and $C$ coincide.

The following categories are used in the sequel: 


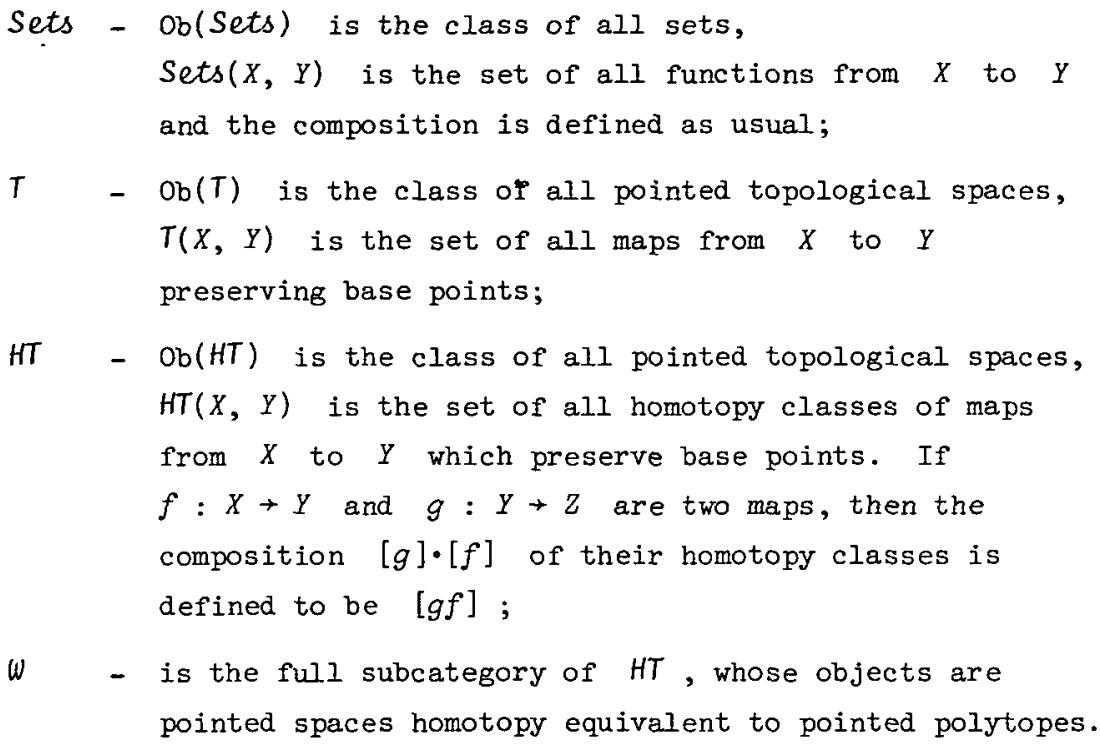

A morphism $f \in \mathcal{C}(X, Y)$ is called an isomorphism if there is $g \in C(Y, X)$ such that $g f=i d_{X}$ and $f g=i d_{Y}$.

Let $C$ and $D$ be categories. A covariant (respectively contravariant) functor $T: C \rightarrow D$ is a pair consisting of a function $T: \mathrm{Ob}(C) \rightarrow \mathrm{Ob}(D)$ and a family of functions $T_{X Y}=T: C(X, Y) . \rightarrow D(T X, T Y)$ (respectively $T: \mathcal{C}(X, Y) \rightarrow \mathcal{D}(T Y, T X)$ ) such that for any pair $X, Y$ of objects of $C, T(g f)=T(g) \cdot T(f) \quad$ (respectively $T(g f)=T(f) \cdot T(g)$ ) and $T\left(i d_{X}\right)=i d_{T X}$.

Let $Y$ be an object of a category $C$. By $C(Y, \cdot)$ (respectively $C(\cdot, Y)$ ) we denote the covariant (respectively contravariant) functor from $C$ to Sets defined in the following way.

For $X \in \mathrm{Ob}(\mathrm{C})$ we set $\mathrm{C}(Y, \cdot)(X)=\mathrm{C}(Y, X)$ (respectively $\mathcal{C}(\cdot, Y)(X)=\mathcal{C}(X, Y))$ and for $f \in \mathcal{C}\left(X, X^{\prime}\right)$ we have $\mathcal{C}(Y, \cdot) f(g)=f g$ for each $g \in \mathcal{C}(Y, X)$ (respectively $C(\cdot, Y) f(g)=g f$ for each $\left.g \in \mathcal{C}\left(X^{\prime}, X\right)\right)$.

Recall that a directed set $(A,<)$ is a pair consisting of a set $A$ equipped with a non-reflexive and transitive relation " $<$ " such that for any two elements $\alpha, \beta \in A$ there exists an element $\gamma$ with $\gamma>\alpha, \beta$. 
An inverse system $\left(X_{\alpha}, p_{\alpha}^{\alpha^{\prime}}, A\right)$ in a category $C$ consists of a set $\left\{x_{\alpha}\right\}_{\alpha \in A}$ of objects of $C$ indexed by a directed set $A$, and of a set of morphisms $p_{\alpha}^{\alpha^{\prime}} \in \mathcal{C}\left(X_{\alpha^{\prime}}, X_{\alpha}\right)$, where $\alpha^{\prime}>\alpha$ such that for $\alpha^{\prime \prime}>\alpha^{\prime}>\alpha$ there is

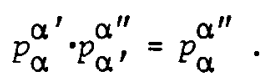

A direct system $\left(X_{\alpha}, p_{\alpha}^{\alpha}, A\right)$ in a category $C$ consists of a set $\left\{x_{\alpha}\right\}_{\alpha \in A}$ of objects of $C$ indexed by a directed set $A$, and of a set of morphisms $p_{\alpha^{\prime}}^{\alpha} \in C\left(X_{\alpha}, X_{\alpha^{\prime}}^{\prime}\right)$, where $\alpha^{\prime}>\alpha$ such that for $\alpha^{\prime \prime}>\alpha^{\prime}>\alpha$ there is

$$
p_{\alpha^{\prime \prime}}^{\alpha^{\prime}} p_{\alpha^{\prime}}^{\alpha}=p_{\alpha^{\prime \prime}}^{\alpha}
$$

If $\left(X_{\alpha}, p_{\alpha}^{\alpha^{\prime}}, A\right)$ is an inverse system in Sets, then $\underset{\alpha}{\lim }\left\{X_{\alpha}, p_{\alpha}^{\alpha^{\prime}}, A\right\}$ is the set of all families $\left\{x_{\alpha}\right\}_{\alpha \in A}$, where $x_{\alpha} \in X_{\alpha}$ and $p_{\alpha}^{\alpha^{\prime}}\left(x_{\alpha^{\prime}}\right)=x_{\alpha}$ for $\alpha^{\prime}>\alpha$.

Suppose $\left(X_{\alpha}, p_{\alpha}^{\alpha}, A\right)$ is a direct system in sets. In the disjoint union of all $X_{\alpha}, \alpha \in A$, we define an equivalence relation " $\sim$ " as follows:

$$
\begin{aligned}
& x_{\alpha} \sim x_{\beta} \text { if and only if there is an index } \delta>\alpha, \beta \text { with } \\
& p_{\delta}^{\alpha}\left(x_{\alpha}\right)=p_{\delta}^{\beta}\left(x_{\beta}\right) .
\end{aligned}
$$

Then $\frac{\lim }{\alpha}\left(X_{\alpha}, p_{\alpha}^{\alpha}, A\right)$ is defined to be the set of all equivalence classes of this relation.

Consider the class of all inverse systems in a category $C$. It is the class of objects of some category, called the pro-category of $\mathcal{C}$ and denoted by pro-C. The set of morphisms in this category is defined by 
(5.1). $\operatorname{pro-C}(X, Y)=\frac{\lim }{\beta} \underset{\alpha}{\lim } \mathcal{C}\left(X_{\alpha}, Y_{\beta}\right)$, if $X=\left\{X, p_{\alpha}^{\alpha^{\prime}}, A\right\}$ and $Y=\left(Y_{\beta}, q_{\beta}^{\beta^{\prime}}, B\right)$

Thus if $B$ is a one-point set, that is, $Y=Y$ is an object of $C$, then

$$
\operatorname{pro} \mathcal{C}(X, Y)=\frac{1 \mathrm{im}}{\alpha}\left(\mathcal{C}(X, Y), \mathcal{C}(\cdot, Y) p_{\alpha}^{\alpha^{\prime}}, A\right) .
$$

Then the composition $g \cdot f$ of two morphisms

$f: X=\left(X_{\alpha}, p_{\alpha}^{\alpha^{\prime}}, A\right) \rightarrow Y \in \mathrm{Ob}(\mathrm{C})$ and $g: Y \rightarrow Z \in \mathrm{Ob}(\mathrm{C})$ of pro-C

is a morphism whose representative is $g \cdot f_{\alpha} \in C\left(X_{\alpha}, z\right)$, where $f_{\alpha} \in c\left(x_{\alpha}, X\right)$ is a representative of $f$ for some $\alpha \in A$.

Now each morphism

$$
f: X=\left\{X_{\alpha}, p_{\alpha}^{\alpha^{\prime}}, A\right\} \rightarrow Y=\left\{Y_{B}, q_{B}^{\beta^{\prime}}, B\right\}
$$

of pro-C can be regarded as a family $\left\{f_{B}\right\}_{B \in B}$, where $f_{B} \in \operatorname{pro} C\left(X, Y_{B}\right)$ and $q_{B}^{\beta^{\prime}} f_{\beta^{\prime}}=f_{\beta}$ for $\beta \leq \beta^{\prime}$.

So we define the composition $g \cdot f$ of two morphisms

$$
\mathbf{f}=\left\{\mathbf{f}_{\beta}\right\}_{\beta \in B}:\left(x_{\alpha}, p_{\alpha}^{\alpha^{\prime}}, A\right) \rightarrow\left(y_{\beta}, q_{\beta}^{\beta^{\prime}}, B\right)
$$

and

$$
\mathbf{g}=\left\{\mathbf{g}_{\delta}\right\}_{\delta \in D}:\left(Y_{B}, q_{B}^{\beta^{\prime}}, B\right) \rightarrow\left(z_{\delta}, r_{\delta}^{\delta^{\prime}}, D\right)
$$

as follows: take for each $\delta \in D$ a representative $g_{\delta}^{\beta(\delta)} \in C\left(Y_{\beta}(\delta), z_{\delta}\right)$ of $g_{\delta}$ and let

$$
g \cdot f=\left\{g_{\delta}^{B(\delta)} \cdot f_{B(\delta)}\right\}_{\delta \in D} .
$$

The identity morphism id $x$ of $x=\left(x_{\alpha}, p_{\alpha}^{\alpha^{\prime}}, A\right)$ is the family $\left\{p_{\alpha}\right\}_{\alpha \in A}$, where the identity morphism $i d_{X_{\alpha}}$ is a representative of 
$\mathrm{p}_{\alpha}: X \rightarrow X_{\alpha}$ for each $\alpha \in A$. The morphism $\mathrm{p}_{\alpha}$ is called a projection morphism.

Observe that each category $\mathcal{C}$ can be considered as a full subcategory of pro-C. It is also clear that any covariant functor $F: \mathcal{C} \rightarrow \mathbb{D}$ induces in a natural way the functor pro- $E: \operatorname{pro}-C \rightarrow$ pro- $D$.

An object $X$ of pro-C is stable if it is isomorphic in pro-C to some object of $C$.

Let $X$ be a topological space. Recall that a family of maps $\pi=\left\{\pi_{j}: X \rightarrow\langle 0,1\rangle\right\}_{j \in\langle 0,1\rangle}$ is a partition of unity if $\sum_{j} \pi_{j}(x)=1$ for every $x \in X$. A covering $U$ of $X$ is numerable if a partition of unity $\pi=\left\{\pi_{U}\right\}_{U \in U}$ exists such that

$$
\pi_{U}^{-1}(0,1) \subset U \text { for every } U \in U
$$

Let $\left(x, x_{0}\right)$ be a pointed topological space and let $\left\{U_{\alpha}\right\}_{\alpha \in A}$ be the set of all locally finite numerable open coverings of $X$ such that each $U_{\alpha}$ has exactly one member containing $x_{0}$. If $U_{\alpha^{\prime}}$ is a refinement of $U_{\alpha}$ and $\alpha^{\prime} \neq \alpha$, then we put $\alpha<\alpha^{\prime}$. For each $\alpha \in A$, let $N\left(U_{\alpha}\right)$ be the nerve of $U_{\alpha}$, that is $N\left(U_{\alpha}\right)$ is the family of all finite sets $\left\{U_{1}, \ldots, U_{k}\right\}$ such that $U_{i} \in U_{\alpha}, i=1, \ldots, k$, and $\bigcap_{i=1}^{k} U_{i} \neq \varnothing$. Then $N\left(U_{\alpha}\right)$ is a simplicial complex and the body of it equipped with the weak topology is denoted by $K_{\alpha}$ (this means that a subset $B$ of $K_{\alpha}$ is closed if and only if the intersection of $B$ and of any closed simplex is closed in $s$ ). For the base point $k_{\alpha}$ of $k_{\alpha}$ we take the vertex corresponding to the unique element of $U_{\alpha}$ containing $x_{0}$.

Then for each $\alpha \in A$, there exists a map

$$
\pi_{\alpha}:\left(X, x_{0}\right) \rightarrow\left(K_{\alpha}, k_{\alpha}\right)
$$

such that $U$ contains the counterimage of the open star of the vertex $U$ (see [37], p. 355). The homotopy class of $\pi_{\alpha}$ is denoted by $p_{\alpha}$. 
Suppose $\beta>\alpha$. We can choose a map $\mu: U_{\beta} \rightarrow U_{\alpha}$ such that $U \subset \mu(U)$ for every $U \in U_{\beta}$. Then there is the unique simplicial map

$$
\mu_{\beta, \alpha}:\left(\dot{K}_{\beta}, k_{\beta}\right) \rightarrow\left(k_{\alpha}, k_{\alpha}\right)
$$

which agrees with $\mu$ on vertices. Its homotopy class is denoted by $p_{\alpha}^{\beta}$. Then $p_{\alpha}^{\beta} \cdot p_{\beta}=p_{\alpha}$ and $p_{\alpha}^{\beta} \cdot p_{\beta}^{\gamma}=p_{\alpha}^{\gamma}$ for $\alpha<\beta<\gamma \quad$ (see [37], pp. 355-356). Consequently $c\left(X, x_{0}\right)=\left(\left(k_{\alpha}, k_{\alpha}\right), p_{\alpha}^{\beta}, A\right)$ is an inverse system in $W$ called the Cech system of $\left(X, x_{0}\right)$. Moreover,

$$
p_{X}=\left\{p_{\alpha}\right\}_{\alpha \in A}:\left(X, x_{0}\right)+\zeta\left(X, x_{0}\right)
$$

is a morphism of pro-HT.

The main property of the morphism $p_{X}$ is expressed by the following theorem (see [43], p. 22).

THEOREM (5.2). Let

$$
p_{X}=\left\{p_{\alpha}\right\}_{\alpha \in A}:\left(X, x_{0}\right) \rightarrow \check{C}\left(X, x_{0}\right)=\left(\left(k_{\alpha}, k_{\alpha}\right), p_{\alpha}^{\beta}, A\right)
$$

be the natural morphism from a pointed topological space $\left(X, x_{0}\right)$ to its Čech system. Then for any map

$$
f:\left(x, x_{0}\right) \rightarrow(K, k) \in O b(w)
$$

there is a map $f_{\alpha}:\left(k_{\alpha}, k_{\alpha}\right) \rightarrow(k, k)$ with $\left[f_{\alpha}\right] \cdot p_{\alpha}=[f]$. If $g, h:\left(k_{\alpha}, k_{\alpha}\right) \rightarrow(K, k) \in \mathrm{Ob}(W)$ are two maps such that $[g] \cdot p_{\alpha}=[h] \cdot p_{\alpha}$, then $[g] \cdot p_{\alpha}^{\beta}=[h] \cdot p_{\alpha}^{\beta}$ for some $\beta \geq \alpha$.

Theorem (5.2) can be easily generalized to the following

THEOREM (5.3). For any morphism $f:\left(X, x_{0}\right)+Y \in \mathrm{Ob}($ pro-(i) there is a unique morphism $g: \zeta\left(X, x_{0}\right) \rightarrow \gamma$ of pro-w such that $g \cdot p_{X}=f$.

Now we can introduce the shape category Sh. Its objects are all pointed topological spaces and for the set of morphisms $\operatorname{Sh}((X, x),(y, y))$ we take pro- $w(\check{C}(X, x), \check{C}(y, y))$ and the composition of morphisms is induced from pro-w. 
The shape functor $S: H T+S h$ is defined as follows:

$S(X, x)=(X, x)$ and if $f:(X, x) \rightarrow(y, y)$ is a homotopy class, then $S(f)$ is the unique morphism from $\check{C}(X, x)$ to $\check{C}(y, y)$ such that

$$
p_{Y} \cdot f=S(f) \cdot p_{X}
$$

If $(X, x),(y, y) \in \mathrm{ob}(S h)$, then

$$
\operatorname{Sh}(X, x)=\operatorname{Sh}(Y, y)
$$

means that $(X, x)$ and $(y, y)$ are isomorphic in $S h$ and $\operatorname{Sh}(X, x) \geq \operatorname{Sh}(y, y)$ means that $(y, y)$ is dominated by $(X, x)$ in $S h$.

Observe that the property of Čech systems stated in Theorem (5.2) was crucial when defining the shape category. In the case when one deals with compact spaces only, we can take, instead of the Cech system, any expansion of a compact space as an inverse limit of ANR's. This is guaranteed by the following theorem (see [73], p. 272).

THEOREM (5.4). Let $Y=\left(Y_{\beta}, q_{\beta}^{\beta^{\prime}}, B\right)$ be an inverse system of compact Hausdorff spaces, $Y$ its topological inverse limit and $q_{\beta}: Y \rightarrow Y_{\beta}$ projection maps. Then for any map $f: Y \rightarrow K \in \mathrm{Ob}(\omega)$ there is a map $f_{\beta}: y_{B} \rightarrow K$ such that $f \simeq f_{B} \cdot q_{B}$. If $g, h: y_{B} \rightarrow K \in \mathrm{Ob}(\omega)$ are two maps such that $g \cdot q_{\beta} \simeq h \cdot q_{\beta}$, then $g \cdot q_{\beta}^{\beta^{\prime}} \simeq h \cdot q_{\beta}^{\beta^{\prime}}$ for some $\beta^{\prime} \geq \beta$.

Using Theorem (5.4), one can prove that the shape functor is continuous when considered on compact Hausdorff spaces, that is:

THEOREM (5.5). If $Y$ is a topological inverse limit of an inverse system $\left(Y_{B}, q_{B}^{\beta^{\prime}}, B\right)$ of compact Hausdorff spaces and $q_{B}: Y \rightarrow Y_{B}$ are projection maps, then

$$
\mathbf{q}=\left\{S\left[q_{\beta}\right]\right\}_{\beta B}: Y \rightarrow\left\{Y_{B}, S\left[q_{B}^{\beta}\right], B\right\}
$$

is an inverse limit of $\left(Y_{B}, S\left[q_{\beta}^{\beta}\right], B\right)$ in the shape category.

\section{Homotopy and homology pro-groups}

In shape theory the role of the homotopy groups $\pi_{n}(X, x)$ introduced 
by $W$. Hurewicz is taken by the homotopy pro-groups pro- $\pi_{n}(X, x)$. It is the inverse system

$$
\left\{\pi_{n}\left(K_{\alpha}, k_{\alpha}\right), \pi_{n}\left(p_{\alpha}^{\beta}\right), A\right\},
$$

where $\left(\left(K_{\alpha}, k_{\alpha}\right), p_{\alpha}^{\beta}, A\right)$ is the Čech system of a pointed topological space $(X, x)$. The corresponding inverse limit $\bar{\pi}_{n}(X, x)$ is the $n$th shape group of $(X, x)$.

Analogously one can define the $n$th homology pro-group pro- $H_{n}(X)$ as the inverse system

$$
\left\{H_{n}\left(K_{\alpha}\right), H_{n}\left(p_{\alpha}^{\beta}\right), A\right\}
$$

The inverse limit of $\operatorname{pro}_{n}(X)$ is the well known $n$th Čech homology group $\breve{H}_{n}(X)$.

For nice spaces, like ANR's, one can replace the homotopy progroups (or homology pro-groups) by shape groups (respectively, Čech homology groups). However, in general much information is lost by passing to the limit and one must consider the whole pro-group as a new and important shape invariant.

Let us show how some theorems on homotopy and homology groups carry over to shape theory.

THEOREM (6.1) (Moszyńska [83], p. 260). Let $f:(X, x) \rightarrow(y, y)$ be a shape morphism of pointed finite-dimensional continua. If $f$ induces isomorphisms of homotopy pro-groups in all dimensions, then $f$ is a shape equivalence.

Theorem (6.1) was the first generalization of the classical theorem due to Whitehead. Later on some stronger results were obtained by Mardešić ([74], [75]) and by Morita [81]. Edwards and Geoghegan [46] and also Dydak [41] have obtained several theorems of this type weakening the condition that both $X$ and $Y$ be finite-dimensional. However this condition cannot be completely omitted. Indeed, Handel and Segal [52] have shown that a certain continuum constructed by Kahn [59] has non-trivial shape, although all of its homotopy pro-groups vanish. An extensive discussion of 
Whitehead's theorem in shape theory the reader can find in [41].

The second result is a generalization of the W. Hurewicz theorem:

THEOREM (6.2) (see [5], p. 39). Let $(X, x)$ be a pointed continuum. If $\operatorname{pro}_{k}(X, x)$ is isomorphic to the trivial group for $k \leq n \quad(n \geq 1)$, then the Hurewica morphism from $\operatorname{pro}_{n+1}(X, x)$ to $\operatorname{pro}_{n+1}(X)$ is an isomorphism.

\section{Fundamental dimension}

One of the most important shape invariants is the fundamental dimension $F d(X)$ of a compactum $X$. It is defined as the smallest of numbers $\operatorname{dim} X^{\prime}$, where $X^{\prime}$ runs through all compacta with $\operatorname{Sh}(X) \leq \operatorname{Sh}\left(X^{\prime}\right)$. The following theorem gives another characterization of $\operatorname{FD}(X)$.

THEOREM (7.1) (Holsztyński; see [84], p. 215). For every compactum $X$ there exists a compactum $Y$ such that $\operatorname{Sh}(X)=\operatorname{Sh}(Y)$ and $\operatorname{Sh}(X)=\operatorname{dim} Y$.

By a remark due to Nowak ([84], p. 216) the inequality $F d(X) \leq n$, where $X$ is a compactum lying in the Hilbert cube $Q$ and $n$ is a nonnegative integer, holds true if and only if $X$ can be homotopically deformed in each of its neighborhoods (in $Q$ ) to a polyhedron of dimension less than or equal to $n$. This enabled Dydak ([39], p. 439) to define the deformation dimension (or shape dimension) def-dim $X$ of any topological space $X$ as the smallest integer $n$ such that any map $f: X \rightarrow K$ from $X$ to a polytope $K$ is homotopic to a map $g$ whose image is contained in the $n$th skeleton $K^{(n)}$ of $K$. Obviously, in the case of compacta, these two notions coincide.

Observe that the fundamental dimension of every compactum with trivial shape is equal to 0 (in particular, $F d(Q)=0$ ). Moreover, if $X$ and $Y$ are compacta, then $\mathrm{Fd}(X \times Y) \leq \mathrm{Fd}(X)+\mathrm{Fd}(Y)$.

A more detailed study of the fundamental dimension of the cartesian product of two compacta requires the use of algebraic methods. In this way, one obtains

THEOREM (7.2) (Nowak, [86], p. 33). If $X$ and $Y$ are compacta with 
$F d(X) \neq 2$ and $\operatorname{dim} Y=n$ such that for every non-trivial abelian group $\underline{\underline{A}}$ the $n$th cohomology group $H^{n}(Y, \underline{\underline{A}})$ is not trivial, then $\mathrm{Fd}(X \times Y)=\mathrm{Fd}(X)+\mathrm{Fd}(Y)$.

By an example due to Nowak ([85], p. 71) the formula

$$
\operatorname{Fd}(X \times Y)=F d(X)+F d(Y)
$$

fails even for polyhedra. In fact, there exist two connected polyhedra $P_{1}$ and $P_{2}$ such that $\mathrm{Fd}\left(P_{1}\right)=\mathrm{Fd}\left(P_{2}\right)=\mathrm{Fd}\left(P_{1} \times P_{2}\right)=3$.

\section{Movability and related shape invariants}

An important shape invariant due to Borsuk is the movability and also the $n$-movability. Let $X$ be a compactum lying in a space $M \in \mathrm{AR}$. One says that $X$ is movable (n-movable), if for every neighborhood $U$ of $X$ in $M$ there exists a neighborhood $V$ of $X$ in $M$ such that for every neighborhood $W$ of $X$ in $U$ and for any map $f: Y \rightarrow V$, here $Y$ is a compactum (respectively $n$-dimensional compactum), there is a homotopy $\varphi: Y \times\langle 0,1\rangle \rightarrow U$ such that

$$
\varphi(y, 0)=f(y) \text { and } \varphi(y, 1) \in W \text { for every } y \in Y .
$$

One shows that the choice of the space $M \in A R$ containing $X$ is immaterial, for both the movability and the $n$-movability. It is clear that every movable compactum is $n$-movable for $n=1,2, \ldots$. Let us add that if $X_{1}, X_{2}, \ldots$ are movable compacta, then the cartesian product $x_{1} \times x_{2} \times \ldots$ is also movable.

Both properties: the movability and the n-movability are monotone shape invariants, that is, the movability of a compactum $X$ (or the $n$-movability of $X$ ) implies the movability (or the $n$-movability, respectively) of every compactum $X^{\prime}$ with $\operatorname{Sh}(X) \geq \operatorname{Sh}\left(X^{\prime}\right)$.

One transfers, in a natural way, the definitions of movability and of $n$-movability onto pointed compacta.

The class of all movable compacta is large. It contains, in particular, all ANR's and also all plane compacta. However in the 3-dimensional euclidean space $E^{3}$ there exist continua which are not movable (even not l-movable); for instance all solenoids of van Dantzig. 
As we have already noticed, there does not exist a compactum with shape greater than or equal to the shapes of all solenoids. Therefore the following theorem is remarkable.

THEOREM (8.1) (Spiez, [95], p. 619). There exists a movable compactum $X_{0}$ such that $\operatorname{sh}\left(X_{0}\right) \geq \operatorname{Sh}(X)$ for every movable compaction $X$.

Thus the class of all movable compacta is in some sense shape-bounded, in contrast to the class of all continua.

One shows that if every component of a compactum $X$ is movable (or $n$-movable), then $X$ is movable (or $n$-movable, respectively). However the converse fails, because there exist movable compacta for which there are several components which are even not movable (see [14], p. 165).

THEOREM (8.2) (Trybulec, [97], p. 732). Every movable curve has the shape of a plane curve.

It follows, that the shape of a movable curve is determined by its first Betti number of it. Consequently the collection of shapes of movable curves is countable.

THEOREM (8.3) (Dydak [40], p. 60). If $(X, x)$ is a 1-movable pointed continum and $\operatorname{Sh}(X)=\operatorname{Sh}(y)$, then $\operatorname{Sh}(X, x)=\operatorname{Sh}(y, y)$ for every $y \in Y$.

So, if we call a continuum $X$ pointed l-movable (pointed movable) when $(X, x)$ is l-movable (movable) for some point $x \in X$, we get in this way some unpointed shape invariants. Here are some properties of these notions.

THEOREM (8.4) (Krasinkiewicz [71], p. 144). If $X$ is movable and pointed 1-movable, then it is pointed movable.

THEOREM (8.5) (Krasinkiewicz [71], p. 152 and McMillan [75]). A continuous image of a pointed 1-movable continum is pointed 1-movable.

THEOREM (8.6) (Krasinkiewicz [72]). A continuou $X$ is pointed 1-movable if and only if it has the shape of a locally connected continum.

Some relations between pointed 1-movability of a continuum $X$ and the existence of so called approximative paths joining two points of $X$ have been recently established by Krasinkiewicz and Minc (see [73]). 
The following problem remains still open. Is every movable continuzm pointed movable?

\section{Shape embedding properties}

Let us assign to every compactum $X \neq \emptyset$ a coefficient $e(X)$ defined as the minimal number $n$ such that the $n$-dimensional euclidean space $E^{n}$ contains a compactum $Y$ with $\operatorname{Sh}(X)=\operatorname{Sh}(Y)$. If such a natural number $n$ does not exist, then we set $e(X)=\infty$. Clearly $e(X)$ is a shape invariant.

A remarkable example given recently by Kadlof ([58], p. 905) shows that $e(X)$ is not a monotone shape invariant, because there exist two polyhedra $P_{1}, P_{2}$ such that $P_{1} \subset E^{3}$ (hence $e\left(P_{1}\right) \leq 3$ ), and $\operatorname{Sh}\left(P_{1}\right)>\operatorname{Sh}\left(P_{2}\right)$, but $e\left(P_{2}\right)>3$.

The following question remains open:

(9.1). Is it true that if $\left(x, x_{0}\right)$ is a pointed continum with trivial group $\pi_{1}\left(X, x_{0}\right)$, then $e(Y) \leq e(X)$ for every continuum $Y$ with $\operatorname{Sh}(Y)<\operatorname{Sh}(X)$ ?

By the well known theorem of $\mathrm{K}$. Menger and G. Nöbeling, every compactum $X$ with dimension less than or equal to $n$ is homeomorphic to a compactum $Y \subset E^{2 n+1}$. Consequently $e(X) \leq 2 n+1$ for every compactum $X$ with $\mathrm{Fd}(X) \leq n$. Moreover, there exists for every natural number $n$, an $n$-dimensional polyhedron $P$ such that no subset of $E^{2 n}$ is homeomorphic to $P$. However, for fundamental dimension, the following problem remains still open.

(9.2). Does there exist, for every natural number $n, a$ compactum $X$ such that $\mathrm{Fd}(X)=n$ and $e(X)=2 n+1$ ?

By a recent result due to Duvall and Hush ([38]), if $n=2^{k}$, then the answer to this question is positive. On the other hand, Ivanšic showed (see [56], p. 473) that every pointed l-movable continuum of dimension $n$ has the shape of a pointed compactum lying in the space $E^{2 n}$. 


\section{Fundamental retractions. Spaces FANR}

Let $X, Y$ be compacta, $Y \subset X \subset M \in A R$. A fundamental sequence $r=\left\{r_{k}, X, Y\right\}_{M, M}$ is said to be a fundamental retraction of $X$ to $Y$ if $r_{k}(y)=y$ for every $y \in Y$ and $k=1,2$. If there exists a fundamental retraction of $X$ to $Y$, then $Y$ is said to be a fundamental retract of $X$. It is clear how to transfer those notions onto the case of pointed compacta.

It is known that every fundamental retraction of $X$ to $Y$ (or of $\left(X, x_{0}\right)$ to $\left.\left(Y, x_{0}\right)\right)$ induces an $r$-homomorphism of the group $\bar{h}_{n}(X, \underline{\underline{A}})$ onto $\breve{H}_{n}(Y, \underline{\underline{A}})$ (and also an r-homomorphism of $\check{\pi}_{n}\left(X, x_{0}\right)$ onto $\check{\pi}_{n}\left(Y, x_{0}\right)$ respectively). One knows also that the movability (or n-movability) passes from $X$ (or from $\left(x, x_{0}\right)$ ) onto their fundamental retracts.

By FANR-sets one understands compacta $X$ such that for every compactum $X^{\prime} \supset X$ there exists a compact neighborhood $U$ of $X$ in $X^{\prime}$ such that $X$ is a fundamental retract of $U$. The concept of FANR-spaces is a shape invariant. Moreover, the following theorem (see [14], p. 254) holds true.

THEOREM (10.1). A compactum $X$ is an FANR-set if and only if it is shape dominated by a polyhedron.

Among plane compacta, the sets $X \in$ FANR are characterized by the finiteness of Betti numbers $p_{0}(X)$ and $p_{1}(X)$.

The class of FANR-spaces constitutes a natural extension of the class of ANR's and the global properties of FANR's are similar to some extent, to the global properties of ANR's, hence also to the global properties of polyhedra. However the similarity with the global properties of polyhedra is limited, because Edwards and Geoghegan (see [45], p. 275) have constructed a 2-dimensional FANR-set which is not of polyhedral shape. But, as it was recently shown by Kadlof, every pointed l-movable FANR-set lying in the space $E^{3}$ has the shape of a polyhedron.

It is still unknown whether every FANR-set has the shape of a polytope. This seems to be a difficult problem. So it makes sense to distinguish the class of compacta (called pointed FANR's) being FANR-sets 
with the shape of polytopes. Let us state some properties of pointed FANR's.

THEOREM (10.2) (Demers [36], p. 4 and Edwards and Geoghegan [45], p. 523). Let $X$ be a continurm. If $(X, x)$ is shape dominated by some pointed polytope, then $(X, x)$ has the shape of a pointed polytope.

THEOREM (10.3) (Geoghegan, [49], p. 278). If $X$ has the shape of a polytope, then $(X, x)$ has the shape of a pointed polytope for every $x \in X$.

THEOREM (10.4) (Dydak, Nowak and Strok, [42], p. 487). If $X, Y$ and $X \cap Y$ are pointed FANR's, then $X \cup X$ is a pointed FANR.

\section{Shape-regular compacta}

It is well known that the shape of every fundamental retract of a compactum $X$ is less than or equal to $\operatorname{Sh}(X)$. Let us say that $X$ is shape-regular, if for every compactum $Y$ with $\operatorname{Sh}(Y) \leq \operatorname{Sh}(X)$ there exists a fundamental retract $Y^{\prime}$ of $X$ such that $\operatorname{Sh}(Y)=\operatorname{Sh}\left(Y^{\prime}\right)$. One sees easily that already in the plane $E^{2}$ there exist continua which are not shape-regular. So it is, for instance, with any continuum $X \subset E^{2}$ which decomposes $E^{2}$ into 3 regions and constitutes the common boundary of those regions. It follows, that the shape-regularity is not a shape invariant. In the space $E^{3}$ there exist even connected polyhedra which are not shape-regular. However one can prove that all locally connected curves, and also all locally connected plane compacta are shape-regular (see [21]).

THEOREM (11.1). If $X \in$ FANR, then $X \times Q$ is shape-reguzar.

The question whether for every compactum $X$ there exists a shaperegular compactum $X^{\prime}$ with $\operatorname{Sh}(X)=\operatorname{Sh}\left(X^{\prime}\right)$ remains open. This question is related to the general problem to select among all compacta with a given shape, a representative with especially interesting topological properties. It is known (see [17], p. 1146) that for every continuum $X$ there exists an indecomposable continuum $X^{\prime}$ with $\operatorname{Sh}\left(X^{\prime}\right)=\operatorname{Sh}(X)$. However, as has been recently shown by Krasinkiewicz there exist continua $X$ with shape different from every hereditary indecomposable continuum. 
To similar problems belongs also the question of giving a system of shape properties characterizing the shapes of all closed manifolds. Recently, Spiez solved this problem in the special case of all 2-dimensional manifolds (that is, of all surfaces).

\section{Some operations on shapes}

One shows easily that for every two compacta $X, Y$ the shape of the cartesian product $X \times Y$ depends only on the shapes of $X$ and $Y$. Thus one can define the operation of the multiplication of shapes by the formula

$$
\operatorname{Sh}(X) \times \operatorname{Sh}(Y)=\operatorname{Sh}(X \times Y) .
$$

The shapes $\operatorname{Sh}(X)$ and $S h(Y)$ are said to be factors of the shape $\operatorname{Sh}(X \times Y)$. Similarly one defines the multiplication of pointed shapes by the formula

$$
\operatorname{Sh}\left(X, x_{0}\right) \times \operatorname{Sh}\left(Y, y_{0}\right)=\operatorname{Sh}\left(X \times Y,\left(x_{0}, y_{0}\right)\right) .
$$

THEOREM (12.1) (Trybulec, [98], p. 69). For every movable continuum $X$ there exists at most one representation of $\operatorname{Sh}(X)$ as a finite or countable product of shapes of curves.

However the question whether in this theorem the hypothesis of the movability is essential, remains open.

The shapes of compacta which can not be decomposed into two not trivial shapes are called prime shapes. By an example due to Sieradzki ([93], p. 97) there exist polyhedra with shape for which there exist two different decompositions into product of prime shapes. Another example of this kind is given in [14], p. 356. It is still unknown if for every compactum $X$ there exists a prine factor of $\operatorname{Sh}(X)$.

There exist many other results and other open questions concerning the decomposition of shapes into factors. In particular, the question remains open if for every $X \in$ FANR there exists only a finite number of factors of $\operatorname{Sh}(X)$. Also the question if $\operatorname{Sh}(X) \times \operatorname{Sh}\left(S^{\mathcal{l}}\right)=\operatorname{Sh}(Y) \times \operatorname{Sh}\left(S^{\mathcal{l}}\right)$ implies that $\operatorname{Sh}(X)=\operatorname{Sh}(Y)$ remains open.

It is clear that for given pointed compacta $\left(x, x_{0}\right),\left(y, y_{0}\right)$ there exist two pointed compacta $\left(X^{\prime}, a\right),\left(y^{\prime}, a\right)$ such that $X^{\prime} \cap Y^{\prime}=\{a\}$ and $\left(X^{\prime}, a\right),\left(Y^{\prime}, a\right)$ are homeomorphic to $\left(X, x_{0}\right)$ and $\left(y, y_{0}\right)$ respectively. 
One sees that the shape of $\left(X^{\prime} \cup Y^{\prime}, a\right)$ depends only on the shapes $\operatorname{Sh}\left(X, x_{0}\right)$ and $\left(y, y_{0}\right)$. Thus one can define the addition of pointed shapes by the formula:

$$
\operatorname{Sh}\left(X, x_{0}\right)+\operatorname{Sh}\left(Y, y_{0}\right)=\operatorname{Sh}\left(X^{\prime} \cup Y^{\prime}, a\right) .
$$

The shapes $\operatorname{Sh}\left(X, x_{0}\right)$ and $\operatorname{Sh}\left(Y, y_{0}\right)$ are said to be constituents of the shape $\operatorname{Sh}\left(X, x_{0}\right)+\operatorname{Sh}\left(Y, y_{0}\right)$. If there does not exist a decomposition of $\operatorname{Sh}\left(X, x_{0}\right)$ into the sum of two not trivial pointed shapes, then we say that $\operatorname{Sh}\left(X, x_{0}\right)$ is simple.

THEOREM (12.1) (see [54], p. 288). If $M$ is a closed, connected $n$-dimensional maniford and $a \in M$, then $\operatorname{Sh}(M, a)$ is simple.

\section{Modified Lusternik-Schnirelmann category}

By the modified Lusternik-Schnirelmann category of a compactum $X$ (lying in an AR-space $M$ ), one understands the number $\boldsymbol{N}(X)$ defined as follows.

If $X=\varnothing$, then $\boldsymbol{\xi}(X)=0$.

If $X \neq \emptyset$ and if there exist natural numbers $n$ such that for every neighborhood $U$ of $X$ (in $M$ ) there are compacta $x_{1}, \ldots, x_{n}$ contractible in $U$ such that $X=X_{1} \cup X_{2} \cup \ldots \cup x_{n}$, then $\boldsymbol{s}(X)$ denotes the smallest of all such numbers $n$.

In all other cases, one sets $\boldsymbol{\aleph}(X)=\infty$.

One proves (see [19], pp. 36 and 37) that the choice of the space $M \in \mathrm{AR}$ containing $X$ is immaterial and that $\boldsymbol{\kappa}(X)$ is a monotonous shape invariant, satisfying the inequality $\boldsymbol{N}(X) \leq \mathrm{Fd}(X)+1$. Moreover, one shows that if $T_{n}$ is the cartesian product of $n$ circles $S^{l}$, then $\boldsymbol{\kappa}\left(T_{n}\right)=n+1$. One knows also (by an example due to Case and Chamberl in [25]) that $\boldsymbol{s}(X)$ is not determined by the homology and the fundamental groups of $X$. Moreover the question whether the same holds true for movable compacta remains open.

Let us assign to every compactum $X$ two coefficients: 
$\alpha(X)$ equals the smallest cardinal number $\underline{\underline{m}}$ such that $X$ is the union of $m$ compacta with trivial shape;

$\alpha(X)$ equals the smallest of all cardinal numbers $\alpha(Y)$, where $y$ runs through all compacta $Y$ with $\operatorname{Sh}(Y) \geq \operatorname{Sh}(X)$.

The question remains open if for every compactum $X$ there is a compactum $Y$ with $\operatorname{Sh}(X)=\operatorname{Sh}(Y)$ with $\alpha(X)=\alpha(Y)$.

It is clear that $1 \leq \alpha(X) \leq 2^{\boldsymbol{K}_{0}}$ for every compactum $X \neq \emptyset$ and that for any compacta $x_{1}, x_{2},\left(x_{1} \cup x_{2}\right) \leq \alpha\left(x_{1}\right)+\alpha\left(x_{2}\right)$ and $\alpha\left(X_{1} \times X_{2}\right) \leq \alpha\left(X_{1}\right)+\alpha\left(X_{2}\right)$.

One shows (see [20]) that $\boldsymbol{\alpha}(X)$ is a monotonous shape invariant and that $\boldsymbol{\alpha}(X) \geq \boldsymbol{\aleph}(X)$ for every compactum $X$. Moreover $\boldsymbol{\alpha}\left(T_{n}\right)=n+1$ and there exist compacta $X$ with $\alpha(X)=\kappa_{0}$. It is known (see [20]) that for every $X \in$ FANR the coefficient $\alpha(X)$ is finite. Moreover, for every plane continuum $X$ the coefficient $\boldsymbol{\alpha}(X)$ is less than or equal to 2 and so is also for every movable curve $X$. The question remains open if there exists a continuum $X \subset E^{3}$ with $4 . \leq \alpha(X) \leq \kappa_{0}$.

Let us add that Dydak and Nowak recently proved that if $X$ is a continuum with $\alpha(X)<2^{\aleph_{0}}$, then $X$ is 1-movable.

\section{Cell-1ike maps}

A map $f: X \rightarrow Y$ of a compactum $X$ into another compactum $Y$ is called cell-like, provided all its point inverses have trivial shapes. The theory of cell-like maps constitutes one of the most interesting parts of geometric topology. We refer the reader to [72] for a survey of it. Let us start with results about cell-like maps whose domains and ranges are locally nice.

THEOREM (14.1) (Lacher [72], p. 510). A map $f: X \rightarrow Y$ where $X, Y \in \mathrm{ANR}$ is cell like if and only if for every open set $V$ of $Y$ the restriction $f / f^{-1}(V): f^{-1}(V) \rightarrow V$ is a homotopy equivalence.

Easy corollaries to Theorem (14.1) are the following:

COROLLARY (14.2). If $f: X \rightarrow Y$ is a cell-like map between ANR's 
and the shape of $B \subset Y$ is trivial, then the shape of $f^{-1}(B)$ is trivial.

COROLLARY (14.3). If $X, Y, Z$ are ARN's and if $f: X \rightarrow Y$ and $g: Y \rightarrow Z$ are cell-like maps, then $g f: X \rightarrow Z$ is a cell like map.

In particular, one gets from Corollary (14.3) that ANR's and cell like maps form a category.

The following result was used by West in proving that ANR's have homotopy type of finite polyhedra (recall that a Q-manifold is a space locally homeomorphic to the Hilbert cube $Q$ ).

THEOREM (14.4) (West [94], p. 12). For every $X \in$ ANR there exists a Q-manifold $M$ and a cell-like map $f: M \rightarrow X$.

Moreover Chapman [26] gave the first proof that simple homotopy type is a topological invariant, and later extended this result to the following

THEOREM (14.5) (Chapman [29], p. 230). A cell-like map between polyhedra is a simple homotopy equivalence.

Concerning the notion of a simple homotopy equivalence see ([100]).

Let us mention that Theorem (14.5) enabled Chapman [29] to extend the simple homotopy theory from the category of finite CW-complexes to the category of all ANR's . More specifically: he calls a homotopy equivalence $f: X \rightarrow Y$ between $\mathrm{ANR}^{2} \mathrm{~s}$ to be a simple homotopy equivalence provided there exists a set $Z \in$ ANR and two cell-like maps $g: Z \rightarrow X$ and $h: Z \rightarrow Y$ such that $f g \simeq h$. Thus cell-like maps between ANR's (polyhedra) have to be homotopy equivalences (simple homotopy equivalences).

Let us mention what is known about cell-like maps between manifolds.

THEOREM (14.6) (Siebenmann [92], p. 271). Let $M$ and $N$ be closed $n$-manifolds $(n \geq 5)$. Then the set of all cell-like maps from $M$ onto $N$ is precisely the closure of the set of homeomorphisms from $M$ onto $N$ in the space of maps from $M$ to $N$.

Theorem (14.6) holds true in case $n=3$ under the additional assumption that $M$ contains no fake 3-cells, that is, compact contractible 3-manifolds not homeomorphic to the standard 3-cell (see [4]). 
The main difference between the theory of cell-like maps of ANR's and the cell-like maps of compacta is that the last ones do not have to be shape equivalences. In fact, Keesling [63], p. 997, constructed a celllike map of the Hilbert cube $Q$ onto a non-movable compactum $Y$ and Taylor [96] gave an example of a cell-like map of a compactum $X$ with nontrivial shape onto $Q$. However, in some cases cell-like maps are shape equivalences.

THEOREM (14.7) (Sher [83], p. 86; see also [8] and [64]). A celZlike map between finite-dimensional compacta is a shape equivalence.

The difficulties with cell-like mups between compacta led Kozlowski [65] to introduce a subclass of the class of all cell-like maps. Namely, he calls a map $f: X \rightarrow Y$ a hereditary shape equivalence provided for any closed subset $B$ of $Y$ the restriction

$$
f / f^{-1}(B): f^{-1}(B) \rightarrow B
$$

is a shape equivalence. By taking one-point sets for $B$ one gets that every hereditary shape equivalence is cell-like.

Using Theorem (14.1), one gets that every cell-like map between ANR's is a hereditary shape equivalence. Theorem (14.7) implies that also every cell-like map between finite-dimensional compacta is a hereditary shape equivalence.

THEOREM (14.8) (Kozlowski, see [43], p. 138). If $f: X \rightarrow Y$ is a cell-like map and the dimension of $y$ is finite, then $f$ is a hereditary shape equivalence.

The main property of hereditary shape equivalences is expressed in the following

THEOREM (14.9) (Kozlowski, [43], p. 133). Let $f: X \rightarrow Y$ be a hereditary shape equivalence. If $X$ is a subset of a compactum $Z \in \mathrm{ANR}$, then $Z u_{f} Y \in$ ANR.

\section{Final remarks}

In this short exposition of the most simple ideas and results of the theory of shape, we have omitted several important topics, which still await their whole development. 
(a) Covering space theory and shape fibrations. The idea of transferring the classical notions of covering space and of overlay theory to the theory of shape is due to Fox (see [48]). Later Coram and Duvall, introduced the notion of approximate fibration, replacing the lifting property basic for the notion of Hurewicz fibration, by the more general approximate homotopy lifting property ([34]). An essential step in this process was taken by Mardešić and Rushing (see [76] and [77]), who have introduced a new class of maps between locally compact metric spaces, called shape fibrations. They coincide with approximative fibrations in the case of ANR's.

(b) Theory of position. It is clear that concepts of the classical theory of knots, as yet developed only for very special classes of closed curves, concern in fact several phenomena of a global character. There are some attempts to introduce a more general notion of position, which will allow us to exhibit some global aspects of the theory of position, in particular, of the theory of knots. For instance, one can show (see [13], p. 155) that every plane continuum $X$ with $p_{1}(X)=1$, can be embedded in the 3-dimensional euclidean space $E^{3}$ so that it constitutes in $E^{3}$ a knot similar (in some sense) to a given polygonal knot. The connection of position with shape theory was studied also by Sher ([91]). One can suppose that many phenomena of position of compacta are intimately related to the shape properties of those compacta and their complement sets.

(c) Another domain which probably has a close connection with the notions of the theory of shape is the endeavour to express the difference between the global properties of two compacta in the terms of topological notions in the collection of all compacta lying in a given space. The well known metric of Hausdorff does not give any account on the relation between the distance of two compacta and their topological properties. There exist some attempts to introduce another metric free from this defect (see [9] and [69]), but limited only to ANR's. Another step in this direction examines metrics in the collection of compact subsets of a given space, by which a relation between the distance of two arbitrary, non-empty compacta and their shape properties is taken into consideration. There are several papers concerning those ideas ([18], [24], [32], [33]). However the researches in this domain are still only at their beginning. 
(d) Proper shape theory, introduced by Ball and Sher (see [7] and also [6]) is an attempt to transfer the concepts of the theory of shape of compacta to the more general case of locally compact spaces. Instead of maps, one considers proper maps (that is, maps $f: X \rightarrow Y$ such that for every compact subset $B$ of $Y$ the set $A=f^{-1}(B) \subset X$ is compact). In this way one gets the notion of the proper shape category and one obtains a theory which in the case of compacta agrees with the usual shape theory and gives a good insight into global properties of locally compact spaces.

(e) Shape properties of topological groups. The important task to study properties of continua which support a topological group structure has been considered, in particular in some papers of Keesling (see [60], $[61],[62])$.

\section{References}

[1] П.С. Аленсандров [P.S. Aleksandrov], "О некоторых основных моментах в нсторни топологни тенущего столетия" [Some of the major moments in the history of topology in the current century], Proceedings of the Topology Conference, Kisyniev, 1979.

[2] П.С. Аленсандров [P.S. Aleksandrov], "Opening Address", Tuрасполоский Симпозиум по Обией Топологии и ее Грилохениям [Proceedings of the Symposium on General Topology and Applications], Moscow 1979, to appear.

[3] П.С. Аленсандров, В.В. Фелорчун [P.S. Aleksandrov and V.V. Fedorchuk], "Основные моменты в развнтии теоретиномнонественной топологии" [The main aspects in the developement of set-theoretical topology], Uspehi Mat. Nauk 33 (1978), 3-48; English Translation: Russian Math. Surveys 33 (1978), 1-53.

[4] Steve Armentrout, Cellular decompositions of 3-manifolds that yield 3-manifolds (Memoirs of the American Mathematical Society, 107. American Mathematical Society, Providence, Rhode Island, 1971).

[5] M. Artin, B. Mazur, Etale homotopy (Lecture Notes in Mathematics, 100. Springer-Verlag, Berlin, Heidelberg, New York, 1969). 
[6] B.J. Ball, "Alternative approaches to proper shape theory", Studies in topology, 1-27 (Proc. Conf., University of North Carolina, Charlotte, North Carolina, 1974. Academic Press, New York, London, 1975).

[7] B.J. Ball and R.B. Sher, "A theory of proper shape for locally compact metric spaces", Fund. Math. 86 (1974-1975), 163-192.

[8] C. Богатый [S. Bogatyi], "О теореме Виеториса в натегорин гомотоний и одной проблеме Борсуна" [The theorem of Vietoris in the homotopy category, and a certain problem of Borsuk], Fund. Math. 84 (1974), 209-228.

[9] K. Borsuk, "On some metrizations of the hyperspace of compact sets", Fund. Math. 41 (1955), 168-202.

[10] Karol Borsuk, Theory of retracts (Monografie Matematyczne 44. PWN - Polish Scientific Publishers, Warszawa, 1967).

[11] Karol Borsuk, "Concerning homotopy properties of compacta", Fund. Math. 62 (1968), 223-254.

[12] K. Borsuk, Theory of shape (Lecture Notes Series, 28. Matematisk Institut, Aarhus Universitet, Aarhus, 1971. Reprinted, 1973).

[13] Karol Borsuk, "On positions of sets in spaces", Fund. Math. 79 (1973), 141-158.

[14] Karol Borsuk, Theory of shape (Monografie Matematyczne 59. PWN Polish Scientific Publishers, Warszawa, 1975).

[15] H. Борсун [K. Borsuk], Teopua Lleunos [Theory of Shape] (Mir, Moscow, 1976).

[16] Karol Borsuk, "On a new shape invariant", Topology Proceedings, Vol. I, 1-9 (Conf., Auburn Univ., Auburn, Ala., 1976. Mathematics Department, Auburn University, Auburn, Alabamba, 1977).

[17] Karol Borsuk, "A remark on shape of continua", Bulz. Acad. Polon. Sci. Sér. Sci. Math. Astronom. Phys. 25 (1977), 1141-1147.

[18] Karol Borsuk, "On a metrization of the hyperspace of a metric space", Fund. Math. 94 (1977), 191-207. 
[19] Karol Borsuk, "On the Lusternik-Schnirelmann category in the theory of shape", Fund. Math. 99 (1978), 35-42.

[20] K. Borsuk, "On decompositions of compacta into compacta of trivial shape", Bull. Acad. Polon. Sci. Sér. Sci. Math. Astronom. Phys. (to appear).

[21] Karol Borsuk, "On shape-regularity of several locally connected continua", Buzl. Acad. Polon. Sci. Sér. Sci. Math. Astronom. Phys. (to appear).

[22] K. Borsuk and W. Holsztyński, "Concerning the ordering of shapes of compacta", Fund. Math. 68 (1970), 107-115.

[23] K. Borsuk and J. Oledzki, "Remark on the shape-domination", BuZz. Acad. Pozon. Sci. Sér. Sci. Math. Astronom. Phys. (to appear).

[24] Laurence Boxer and R.B. Sher, "Borsuk's fundamental metric and shape domination", Bulz. Acad. Polon. Sci. Sér. Sci. Math. Astronom. Phys. 26 (1978), 849-853.

[25] J.H. Case and R.E. Chamberlin, "Characterizations on tree-like continua", Pacific J. Math. 10 (1960), 73-84.

[26] T.A. Chapman, "On some applications of infinite-dimensional manifolds to the theory of shape", Furd. Math. 76 (1972), 181-193.

[27] T.A. Chapman, "Shapes of finite-dimensional compacta", Fund. Math. 76 (1972), 261-276.

[28] T.A. Chapman, "Compact Hilbert cube manifolds and the invariance of Whitehead torsion", Bul2. Amer. Math. Soc. 79 (1973), 52-56.

[29] T.A. Chapman, "Cell-like mappings", Algebraic and geometric methods in topology, 230-240 (Conf. Topological Methods in Algebraic Topology, State University of New York, Binghamton, 1973. Lecture Notes in Mathematics, 428. Springer-Verlag, Berlin, Heidelberg, New York, 1974).

[30] T.A. Chapman, "Simple homotopy theory for ANR's ", General Topology App 2. 7 (1977), 165-174. 
[31] Zvonko Čerin, "Shape fibrations, F-stability and FR-stability", Buzz. Acad. Polon. Sci. Sér. Sci. Math. Astronom. Phys. 27 (1979), 417-423.

[32] Zvonko Čerin, "e-calmly regular convergence", submitted.

[33] Zvonko Čerin and A.P. Sostak, "Some remarks on Borsuk's fundamental metric", submitted.

[34] D.S. Coram and P.F. Duvall, Jr., "Approximate fibrations", Rocky Mountain J. Math. 7 (1977), 275-288.

[35] D. van Dantzig, "Ueber topologisch homogene Kontinua", Fund. Math. 15 (1930), 102-125.

[36] Luc Demers, "On spaces which have the shape of C.W.-complexes", Fund. Math. 90 (1975-1976), 1-9.

[37] A. Dold, Lectures on algebraic topology (Die Grundlehren der mathematischen Wissenschaften, 200. Springer-Verlag, Berlin, Heidelberg, New York, 1972).

[38] P.F. Duvall, Jr. and L.S. Hush, "A continuum of dimension $n$ which does not embed up to shape in 2n-space", Proc. Internat. Conf. Geometric Topology, Warsaw 1978 (to appear).

[39] J. Dydak, "Some remarks concerning the whitehead theorem in shape theory", Buzz. Acad. Polon. Sci. Sér. Sci. Math. Astronom. Phys. 23 (1975), 437-445.

[40] Jerzy Dydak, "A simple proof that pointed FANR-spaces are regular fundamental retracts of ANR's ", Bull. Acad. Polon. Sci. Sér. Sci. Math. Astronom. Phys. 25 (1977), 55-62.

[41] Jerzy Dydak, "The Whitehead and Smal theorems in shape theory", Dissertationes Math. (Rozprawy Mat.) 156 (1979), 1-50.

[42] J. Dydak, S. Nowak and M. Strok, "On the union of two FANR-sets", Buzz. Acad. Polon. Sci. Sér. Sci. Math. Astronom. Phys. 24 (1976), 485-489.

[43] Jerzy Dydak, Jack Segal, Shape theory. An introduction (Lecture Notes in Mathematics, 688. Springer-Verlag, Berlin, Heidelberg, New York, 1978). 
[44] David A. Edwards and Ross Geoghegan, "The stability problem in shape, and a Whitehead theorem in pro-homotopy", Trans. Amer. Math. Soc. 214 (1975), 261-277.

[45] David A. Edwards and Ross Geoghegan, "Shapes of complexes, ends of manifolds, homotopy limits and the Wall obstruction", Arm. of Math. (2) 101 (1975), 52l-535.

[46] David A. Edwards and Ross Geoghegan, "Infinite-dimensional Whitehead and Vietoris theorems in shape and pro-homotopy", Trans. Amer. Math. Soc. 219 (1976), 351-360.

[47] Ryszard Engelking, Dimension theory (PWN - Polish Scientific Publishers, Warszawa; North Holland, Amsterdam, Oxford, New York; 1978).

[48] Ralph H. Fox, "On shape", Fund. Math. 74 (1972), 47-71.

[49] Ross Geoghegan, "Elementary proofs of stability theorems in prohomotopy and shape", General Topology Appl. 8 (1978), 265-281.

[50] Ross Geoghegan and R. Richard Summerhill, "Concerning the shapes of finite-dimensional compacta", Trans. Amer. Math. Soc. 179 (1973), 281-292.

[51] Alexander Grothendieck, "Technique de descente et théorèmes d'existence en géométrie algébrique. II: Le théorème d'existence en théorie formelle des modules", Seminaire Bourbaki, 12e année, 1959/60, Fasc. 2, Exp. 195 (Secrétariat Mathématique, Paris, 1960).

[52] D. Handel and J. Segal, "An acyclic continuum with non-movable suspensions", Buzl. Acad. Polon. Sci. Sér. Sci. Math. Astronom. Phys. 21 (1973), 171-172.

[53] Olof Hanner, "Some theorems on absolute neighborhood retracts", Ark. Mat. 1 (1952), 389-408.

[54] John G. Holl ingsworth and Richard B. Sher, "Closed manifolds are of simple shape", Bulz. Acad. Polon. Sci. Sér. Sci. Math. Astronom. Phys. 26 (1978), 287-290.

[55] W. Holsztyński, "An extension and axiomatic characterization of Borsuk's theory of shape", Fund. Math. 70 (1971), 157-168. 
[56] Ivan Ivanšić, "Embedding compacta up to shape", Buzz. Acad. Polon. Sci. Sér. Sci. Math. Astronom. Phys. 25 (1977), 471-475.

[57] A. Kadlof, "Remarks on Borsuk's problems concerning the operation of the addition of pointed shapes", Buzl. Acad. Polon. Sci. Sér. Sci. Math. Astronom. Phys. 24 (1976), 1001-1006.

[58] Andrzej Kadlof, "An example resolving Borsuk's problem concerning the index $e(X)$ ", Bulz. Acad. Polon. Sci. Sér. Sci. Math. Astronom. Phys. 26 (1978), 905-907.

[59] Daniel S. Kahn, "An example in Čech cohomology", Proc. Amer. Math. Soc. $16(1965), 584$.

[60] James Keesling, "On the shape of torus-like continua and compact connected topological groups", Proc. Amer. Math. Soc. 40 (1973), 297-302.

[61] James Keesling, "An algebraic property of the Čech cohomology groups which provents local connectivity and movability", Trans. Amer. Math. Soc. 190 (1974), 151-162.

[62] James Keesling, "Shape theory and compact connected abelian topological groups", Trans. Amer. Math. Soc. 194 (1974), 349-358.

[63] J. Keesling, "A non-movable trivial-shape decomposition of the Hilbert cube", Bulz. Acad. Polon. Sci. Sér. Sci. Math. Astronom. Phys. 23 (1975), 997-998.

[64] Yukihiro Kodama, "On the shape of decomposition spaces", J. Math. Soc. Japan 26 (1974), 636-646.

[65] George Kozlowski, "Images of ANR's ", Trans. Amer. Math. Soc. (to appear).

[66] Józef Krasinkiewicz, "Local connectedness and pointed 1-movability", Bulz. Acad. Polon. Sci. Sér. Sci. Math. Astronom. Phys. 25

[67] J. Krasinkiewicz, "Continuous images of continua and 1-movability", Fund. Math. 98 (1978), 141-164.

[68] Józef Krasinkiewicz and Piotr Minc, "Generalized paths and pointed 1-movability", Fund. Math. 104 (1979), 141-153. 
[69] K. Kuratowski, "Sur une méthode de métrisation complète de certains espaces d'ensembles compacts", Fund. Math. 43 (1956), 114-138.

[10] K. Kuratowski, Topology. Volume I (translated from the French by J. Jaworowski. Academic Press, New York, London; Państwowe Wydawnictwo Naukowe, Warsaw; 1966).

See also: Н. Нуратовсний, Топология. Tom I (translated from the English by M.Ja. AntonovskiT. Izdat. "Mir", Moscow, 1966).

[71] K. Kuratowski, Topology. Volume II (translated from the French by A. Kirkor. Academic Press, New York, London; Panstwowe Wydawnictwo Naukowe [Polish Scientific Publishers], Warsaw; 1968).

See also: H. Нуратовсний, Топология. Tom II (translated from the English by M.Ja. AntonovskiY. Izdat. "Mir", Moscow, 1969).

[72] R.C. Lacher, "Cell-like mappings and their generalizations", BulZ. Amer. Math. Soc. 83 (1977), 495-552.

[73] Sibe Mardešić, "Shapes for topological spaces", General Topology Appl. 3 (1973), 265-282.

[74] Sibe Mardešić, "On the Whitehead theorem in shape theory I", Fund. Math. 91 (1976), 51-64.

[75] Sibe Mardešić, "On the Whitehead theorem in shape theory II", Fund. Math. 91 (1976), 93-103.

[76] Sibe Mardešić, T.B. Rushing, "Shape fibrations I", General Topology Appl. 9 (1978), 193-215.

[17] Sibe Mardě̌ić, T.B. Rushing, "Shape fibrations II", General Topology Appl. (to appear).

[78] Sibe Mardešic and Jack Segal, "Shapes of compacta and ANR-systems", Fund. Math. 72 (1971), 41-59.

[79] Sibe Mardešić and Jack Segal, "Equivalence of the Borsuk and the ANR-system approach to shapes", Fund. Math. 72 (1971), 61-68.

[80] D.R. McMillan, Jr., "One-dimensional shape properties and threemanifolds", Studies in topology, 367-381 (Proc. Conf. Univ. North Carolina, Charlotte, North Carolina, 1974. Academic Press, New York, 1975). 
[81] Ki iti Morita, "The Hurewicz and the Whitehead theorems in shape theory", Sci. Rep. Tokyo Kyoiku Daigaku Sect. A 12 (1974), $264-258$.

[82] Ki iti Morita, "On shapes of topological spaces", Fund. Math. 86 (1975), 251-259.

[83] M. Moszyńska, "The Whitehead theorem in the theory of shape", Fund. Math. 80 (1975), 221-263.

[84] Slawomir Nowak, "Some properties of fundamental dimension", Fund. Math. 85 (1974), 211-227.

[85] Slawomir Nowak, "On the fundamental dimension of approximatively l-connected compacta", Fund. Math. 89 (1975), 61-79.

[86] Slawomir Nowak, "Some remarks concerning the fundamental dimension of the cartesian product of two compacta", Fund. Math. 103 (1979), 3I-4I.

[87] Hanna Patkowska, "A homotopy extension theorem for fundamental sequences", Fund. Math. 64 (1969), 87-89.

[88] Richard B. Sher, "Realizing cell-like maps in euclidean space", General Topology Appl. 2 (1972), 75-89.

[89] R.B. Sher, "Property $S U V^{\infty}$ and proper shape theory", Trans. Amer. Math. Soc. $190(1974), 345-356$.

[90] R.B. Sher, "Proper shape theory and neighborhoods of sets in Q-manifolds", Bulz. Acad. Polon. Sci. Ser. Sci. Math. Astronom. Phys. 23 (1975), 271-276.

[91] R.B. Sher, "Some alternative notions of position", Proc. Internat. Conf. Geometric Topology, Warsaw 1978 (to appear).

[92] L.C. Siebenmann, "Approximating cellular maps with homeomorphisms", Topology 11 (1972), 271-294.

[93] Allan J. Sieradzki, "Non-uniqueness of homotopy factorizations into irreducible polyhedra", Fund. Math. 72 (1971), 97-99.

[94] S. Spiez, "On a plane compactum with the maximal shape", Fund. Math. 78 (1973), 145-156. 
[95] S. Spiez, "A majorant for the family of all movable shapes", Bull. Acad. Polon. Sci. Sêr. Sci. Math. Astronom. Phys. 21 (1973), 615-620.

[96] Joseph L. Taylor, "A counterexample in shape theory", Bul2. Amer. Math. Soc. 81 (1975), 629-632.

[97] A. Trybulec, "On shapes of movable curves", Bulz. Acad. Polon. Sci. Ser. Sci. Math. Astronom. Phys. 21 (1973), 727-733.

[98] A. Trybulec, "On the uniqueness of the decomposition of movable shapes into a product of 1-dimensional shapes", BuZZ. Acad. Polon. Sci. Sér. Sci. Math. Astronom. Phys. 23 (1975), 69-73.

[99] James E. West, "Mapping Hilbert cube manifolds to ANR's : a solution of a conjecture of Borsuk", Ann. of Math. (2) 106 (1977), 1-18.

[100] J.H.C. Whitehead, "Simplicial spaces, nucleii and m-groups", Proc. London Math. Soc. (2) 45 (1939), 243-327.

[101] J.H.C. Whitehead, "On the homotopy type of ANR's ", BuzZ. Amer. Math. Soc. 54 (1948), 1133-1245.

ul Filtrowa $63 \mathrm{~m} 18$,

02-056 Warsaw,

Poland;

ul Sulmierzycka $6 \mathrm{~m} 62$,

02-139 Warsaw,

Poland. 